\title{
Statistical study of particle flux footprint widths with tungsten divertor in EAST
}

\author{
Liu, X.; Naulin, V.; Xu, J. C.; Deng, G. Z.; Rasmussen, J. J.; Liu, J. B.; Nielsen, A. H.; Goldston, R. J.; \\ Maingi, R.; Liu, Y.
}

Total number of authors:

14

Published in:

Plasma Physics and Controlled Fusion

Link to article, DOI:

10.1088/1361-6587/aaf762

Publication date:

2019

Document Version

Peer reviewed version

Link back to DTU Orbit

Citation (APA):

Liu, X., Naulin, V., Xu, J. C., Deng, G. Z., Rasmussen, J. J., Liu, J. B., Nielsen, A. H., Goldston, R. J., Maingi, R., Liu, Y., Xu, G. S., Wang, L., Li, J., \& EAST Team (2019). Statistical study of particle flux footprint widths with tungsten divertor in EAST. Plasma Physics and Controlled Fusion, 61(4), [045001]. https://doi.org/10.1088/1361. $6587 /$ aaf762

\section{General rights}

Copyright and moral rights for the publications made accessible in the public portal are retained by the authors and/or other copyright owners and it is a condition of accessing publications that users recognise and abide by the legal requirements associated with these rights.

- Users may download and print one copy of any publication from the public portal for the purpose of private study or research.

- You may not further distribute the material or use it for any profit-making activity or commercial gain

- You may freely distribute the URL identifying the publication in the public portal 


\title{
Statistical Study of Particle Flux Footprint Widths with Tungsten Divertor in EAST
}

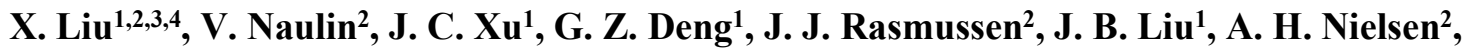 \\ R. Goldston ${ }^{5}$, R. Maingi ${ }^{5}$, Y. Liu ${ }^{1}$, G. S. Xu ${ }^{1}$, L. Wang ${ }^{1, *}$, J. Li ${ }^{1, *}$ and the EAST Team ${ }^{* *}$ \\ ${ }^{1}$ Institute of Plasma Physics, Chinese Academy of Sciences, Hefei 230031, People's Republic of China \\ ${ }^{2}$ Department of Physics, Technical University of Denmark, Kongens Lyngby 2800, Denmark \\ ${ }^{3}$ Sino-Danish College, University of Chinese Academy of Sciences, Beijing 101408, P. R. China \\ ${ }^{4}$ Sino-Danish Center for Education and Research, Beijing 101408, P. R. China \\ ${ }^{5}$ Princeton Plasma Physics Laboratory, Princeton, NJ 08543, USA \\ *E-mail: j_li@ipp.ac.cn and lwang@ipp.ac.cn
}

${ }^{* *}$ See the appendix of B. N. Wan et al., Nucl. Fusion 57, 102019 (2017)

\begin{abstract}
The study of the divertor heat flux is of great importance for the operation of ITER and future fusion devices. In this paper, the behaviors of the widths of the particle flux footprints on the tungsten divertor in EAST have been statistically studied for the first time. A large-number of divertor Langmuir probe data from EAST general discharges in the 2016 campaign have been stepwise selected and analyzed for both L- and H-mode plasmas. It is found that the scaling dependences on poloidal magnetic field for the particle flux fall-off width at the inner tungsten divertor agree well with those at the outer graphite divertor in both L- and H-mode plasmas. The difficulty of extracting reliable footprint widths has been overcome by fitting data from two divertor Langmuir probe arrays distributed toroidally. The nonlinear regressions of the particle flux fall-off width and particle flux spreading width at the inner tungsten divertor in H-mode plasmas have then been performed. A negative dependence on the plasma stored energy is found in the regression of the particle flux fall-off width and the exponent of the stored energy in the regression is consistent with previous experimental and numerical studies. The comparison between the particle flux fall-off width at the tungsten divertor with that at the graphite divertor indicates that statistically the divertor material and the direction of the toroidal magnetic field seem to have no significant influence on the particle flux fall-off width at the inner divertor. In addition, the similar particle flux fall-off width in L- and H-mode plasmas and the in-out asymmetry of the particle flux falloff width at the graphite divertor have also been investigated.
\end{abstract}

Keywords: particle flux footprint widths, tungsten divertor, scrape-off layer, EAST 


\section{Introduction}

One of the major issues that limits machine operations for present tokamaks and future fusion devices is the excessive heat load on the plasma facing components, especially on the divertor targets [1]. The power fall-off width, $\lambda_{q}$, in the scrape-off layer (SOL) is an important parameter to characterize the heat deposition on the divertor target plates. A number of experimental studies have been carried out to scale $\lambda_{q}$ by diagnostics like infrared (IR) camera, midplane reciprocating Langmuir probes, divertor Langmuir probes (Div-LPs) and so on since 1990s [29]. Based on the IR camera data from multiple conventional and spherical tokamaks, Eich et al [5] worked out a multi-machine scaling of $\lambda_{q}$. When scaled to the ITER baseline inductive Hmode scenario, it yields $\lambda_{q, I T E R} \approx 1 \mathrm{~mm}$, much smaller than the value $\lambda_{q, I T E R} \approx 5 \mathrm{~mm}$ in the ITER physics basis [1], which poses a very critical problem. In EAST, dedicated experiments were also conducted with the graphite (C) divertor [6, 7]. From the results of the $\lambda_{q}$ scaling against the poloidal magnetic field $B_{p}$ for both L- and H-mode plasmas, the amplitude of $\lambda_{q}$ in EAST is about two times larger than that in the Eich scaling. Current explanation for this difference is the broadening effect induced by the radio-frequency (RF) heating [10,11]. In addition to the most significant inverse dependence on $B_{p}$ (or plasma current $I_{p}$ ) for the $\lambda_{q}$ scaling, recent works show that $\lambda_{q}$ has negative dependences on the edge electron temperature $T_{e, e d g e}$ for stiff pressure profiles and the main ion mass $A\left(\lambda_{q} \propto A^{-0.15} T_{e, e d g e}^{-0.93}\right)$ [8] and a positive dependence on the divertor leg length in L-mode plasmas [9] and exhibits differently from the inner and outer divertor measurements $[9,12]$.

On the theory side, different models [13-15] and simulation codes [16-22] have been derived/employed to understand the underlying physics of the $\lambda_{q}$ scaling. The heuristic driftbased model proposed by Goldston [13] is very successful to explain the Eich scaling and gives insight on the scaling difference of $\lambda_{q}$ between the inner and outer divertors $[12,23]$. In the heuristic drift-based model, the ion magnetic drifts determine SOL width in the order of the poloidal ion gyro-radius and the radial anomalous electron thermal transport fills the particle channel emptied by the parallel electron thermal conduction [13]. The 6-field 2-fluid 3D turbulence model [24] implemented in BOUT++ framework [25] reproduced the inverse scaling against $I_{p}$ based on discharges in EAST [19] and in C-Mod [20]. In the simulation of EAST discharges, divertor heat flux is found to be dominated by the anomalous electron transport. Different from the heuristic drift-based model, where the ion magnetic drifts play an important role, the neoclassical and turbulent transports are dominant in the EAST simulations [19]. The 2D turbulence code, HESEL, also reproduced the experimental $\lambda_{q}$ scaling in ASDEX-Upgrade (AUG) high collisional L-mode plasmas and reported that the electron conduction and the 
electron and ion advections dominate the parallel heat flux in the near and far SOL, respectively [21]. By scanning physical parameters at the last closed flux surface (LCFS) at the outer midplane (OMP), Olsen et al [21] found a negative dependence on the edge electron temperature and a positive dependence on the edge electron density in the $\lambda_{q}$ scaling [21]. Among all the simulation results, the most astonishing one is from Ref. [22]. Utilizing the gyrokinetic code, XGC1, the experimental results in DIII-D, C-Mod and NSTX, satisfying the Eich scaling, are reproduced and the divertor heat flux is found to be dominated by the ion magnetic drifts in low $I_{p}$ plasma, while by the electron blobby turbulence in high $I_{p}$ plasma [22]. Using the same code, Chang et al [22] predicted $\lambda_{q, I T E R} \approx 5.9 \mathrm{~mm}$ for ITER (blob-dominant), which is about six times the prediction by the Eich scaling. The reason for this much larger $\lambda_{q}$ is explained to be that machine like ITER with larger size has a longer radial scale length of magnetic shear and a wider $\mathrm{E} \times \mathrm{B}$ shear flow layer, which leads to a much greater turbulence spreading of the electron heat flux fall-off width. The explanation indicates that there might be a missing size parameter in the Eich scaling [22]. More experimental data are needed to understand the underling physics clearly.

The upper divertor of EAST was successfully upgraded into the tungsten (W) structure in 2014, which makes it possible to investigate the $\lambda_{q}$ behavior with the $\mathrm{W}$ divertor, in addition to the previous experimental studies of $\lambda_{q}$ in EAST reported with the $\mathrm{C}$ divertor $[6,7,11]$. Additionally, a limited-number and dedicated discharges rather than a large-number of general discharges were used for previous $\lambda_{q}$ investigations. In this paper, the results of statistical study on the divertor particle flux footprint widths (particle flux fall-off width $\lambda_{j s}$ and particle flux spreading width $S_{j s}$ ) are presented, by filtering and analyzing the upper Div-LP data in the 2016 (W divertor) and 2012 (C divertor) experimental campaigns. We mainly focus on analyzing the W-divertor operations in the 2016 campaign. The data in the 2012 campaign are selected to compare the results of the $\mathrm{C}$ divertor with the results of the $\mathrm{W}$ divertor in the 2016 campaign.

The reason to use the Div-LP data instead of the IR camera measurement is due to the fact that, for IR camera the reflection from the $\mathrm{W}$ divertor is difficult to remove in EAST and thus the data processing technique is much more complex than that for Div-LPs. On the other hand, there are also two disadvantages of using Div-LPs. One is the limited poloidal spatial resolution, which is partially solved by combining the data acquired from two toroidal arrays of triple DivLPs at different toroidal locations separated by $\Delta \phi=112.5^{\circ}[26]$. The other is the poor electron temperature measurement, which makes us use $\lambda_{j s}$ to approximate $\lambda_{q}$, which is reasonable for $\mathrm{H}$ mode plasmas (see section 3.3). The rest of this paper is organized as follows. Section 2 introduces the experiment conditions, the method to determine particle flux footprint widths and 
the process to construct the database for analyzing. Section 3 presents the results and discussions of the $\lambda_{j s}$ and $S_{j s}$ scalings for the W divertor, the comparison of the scalings between the particle flux and heat flux footprint widths, the comparison of the W-divertor $\lambda_{j s}$ with the $\mathrm{C}$ divertor $\lambda_{j s}$ and the in-out asymmetry of $\lambda_{j s}$ for the $\mathrm{C}$ divertor. Finally, the results are summarized and concluded in section 4 .

\section{Experiment conditions and database construction}

\subsection{Experiment conditions and diagnostic}

EAST is a fully superconducting tokamak which has a major radius $R \approx 1.85 \mathrm{~m}$, minor radius $a$ $\approx 0.45 \mathrm{~m}$, maximum toroidal magnetic field $B_{t} \approx 3.5 \mathrm{~T}$ and maximum plasma current $I_{p} \approx 1 \mathrm{MA}$ [27]. It equips with $10 \mathrm{MW}$ lower hybrid wave (LHW) current drive system, $1 \mathrm{MW}$ electron cyclotron resonance heating (ECRH) system, $8 \mathrm{MW}$ neutral beam injection (NBI) system and $12 \mathrm{MW}$ ion cyclotron resonance frequency (ICRF) heating system (refer to figure 1(a) for their toroidal locations) [28]. The upper divertor was symmetric to the lower divertor before 2014 and the material of both divertors was $\mathrm{C}$. The upper divertor has been upgraded to the ITER-like actively cooled $\mathrm{W} / \mathrm{Cu}$ monoblock structure consisting of 80 modules and has the capability of steady-state heat removal up to $10 \mathrm{MWm}^{-2}$ since 2014 [29]. Benefiting from this high heat removal capability, the lower divertor is planned to be upgraded to $\mathrm{W}$ in 2019 [30], together with the enhanced particle exhaust capability for the long pulse operations. In this paper, the discharges with the upper single null (USN) configuration in the 2016 and 2012 campaigns are analyzed, with the target material of the divertor being $\mathrm{W}$ and $\mathrm{C}$, respectively.

One of the major missions of EAST is to explore the long-pulse high-performance operations [31], with an H-mode discharge over 100s has been successfully achieved in 2017 [32]. Because of the exploration of non-inductive, purely RF-heated long-pulse H-mode scenario with $\mathrm{W}$ divertor in the 2016 campaign, most of the discharges were USN configured ELMy H-mode (type-I or type-III) heated by LHW, ECRH and/or ICRF. In the 2012 campaign, most of the discharges were L-mode with the lower single null or double null (DN) configuration and heated by LHW and/or ICRF. In our final constructed database, all discharges were configured to USN and $B_{t}$ was configured to the forward ( the ion $\mathrm{B} \times \nabla \mathrm{B}$-drift directed downwards) and reversed directions ( the ion B $\times \nabla B-d r i f t$ directed upwards) in the 2016 (W) and 2012 (C) campaigns, respectively. Normally, the wall was coated with lithium and the divertor was in attached condition in the discharges employed in this paper. 
For reasons mentioned in the introduction, the mainly used diagnostic in this paper is the upper Div-LPs. Two arrays of Div-LPs were installed in the upper inner (UI) and upper outer (UO) divertor targets located at ports D-G in the 2012 campaign [33]. After the upgrade of the upper divertor to $\mathrm{W}$ in the 2014 campaign, the upper Div-LPs are extended to four arrays [26]. These four Div-LP arrays are located at, the upper inner D port (UI-D), upper outer D port (UOD), upper inner O port (UI-O) and upper outer O port (UO-O) divertor targets (see figure 1). Ports $\mathrm{D}$ and $\mathrm{O}$ are separated with the toroidal displacement of $112.5^{\circ}$. In the 2012 campaign, the poloidal spatial resolution of the UI and UO Div-LP arrays was $15 \mathrm{~mm}$ and $\approx 10 \mathrm{~mm}$, respectively. While in the 2016 campaign, the poloidal spatial resolution is $12.5 \mathrm{~mm}$ near the strike point and $18 \mathrm{~mm}$ in the region far from the strike point (limited by the water-cooling system of the monoblock structure), for both the inner and outer Div-LP arrays. In each Div-LP array, triple probes are employed to measure the ion saturation current, the electron density, the electron temperature, the heat flux and so on.

(a)

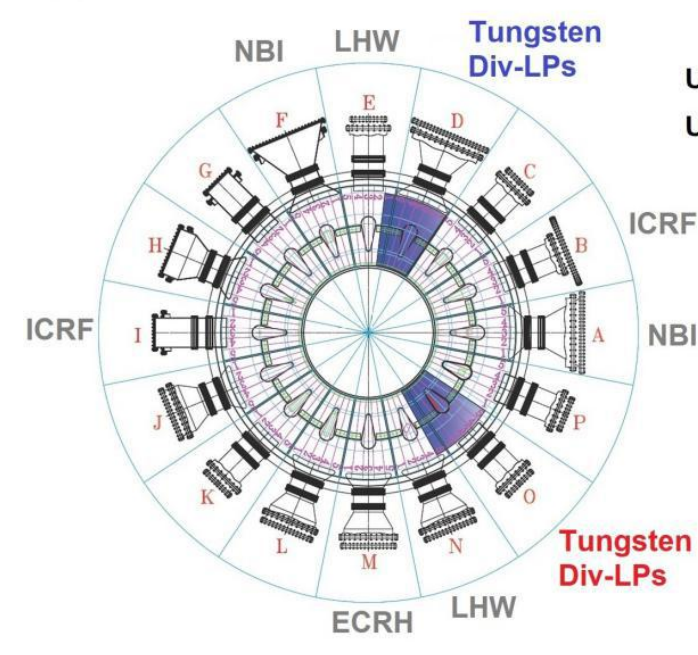

(b)

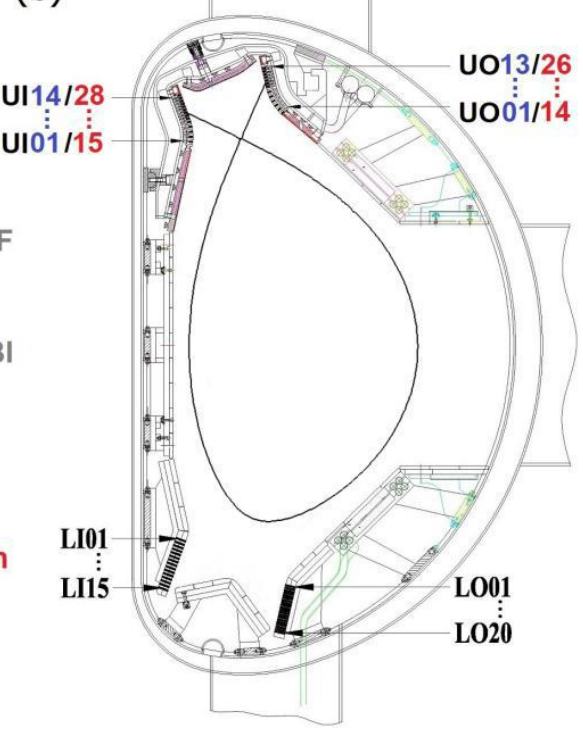

Figure 1. (a) The bird view of the installation locations of the auxiliary heating systems and the upper Div-LPs in 2016. (b) The cross-section view of the poloidal layout of DivLPs in 2016, where blue and red refer to the Div-LP arrays located at port D and port O, respectively.

\subsection{Determination of divertor particle flux footprint widths}

The method to determine divertor particle flux footprint widths is the same as Ref. [5]. Assuming that the profile of the divertor particle flux $\left(\Gamma=j_{s} / e\right.$, where $j_{s}$ is the ion saturation 
current and $e$ is the elementary charge) mapped to the OMP without the radial diffusion into the private flux region (PFR) is exponentially decaying,

$$
\Gamma(r)=\Gamma_{0} \exp \left(-\frac{r}{\lambda_{j s}}\right),
$$

where $\Gamma_{0}$ is the mapped particle flux at the LCFS, $r \equiv R-R_{L C F S}$ is the distance to the LCFS along

the major radius. When a Gaussian function $\left(1 / \pi S_{j s}\right) \exp \left(-r^{2} / S_{j s}^{2}\right)$ is introduced to describe the radial diffusion into the PFR $[4,5]$, the mapped profile of the divertor particle flux becomes the convolution of Eq. (1) and this Gaussian function [5],

$$
\Gamma(r)=\frac{\Gamma_{0}}{2} \exp \left(\left(\frac{S_{j s}}{2 \lambda_{j s}}\right)^{2}-\frac{r-r_{0}}{\lambda_{j s}}\right) \times \operatorname{erfc}\left(\frac{S_{j s}}{2 \lambda_{j s}}-\frac{r-r_{0}}{S_{j s}}\right)+\Gamma_{B G},
$$

where, $r_{0}$ and $\Gamma_{B G}$ are the LCFS location and the background particle flux, respectively. The mapping process is carried out through the magnetic flux equilibrium reconstructed by EFIT. In some studies, Eq. (1) is used to fit only the SOL region of a mapped profile. However, it is more practical to use Eq. (2) in this paper to reduce the fitting uncertainty, because $S_{j s}$ is comparable to, or sometimes even larger than $\lambda_{j s}$ and normally there are not enough points in the SOL region of the measured particle flux profile (see figure 4(b)).

Over $50 \mathrm{~ms}$, all mapped profiles are concatenated together to be a so-called concatenated profile and then fitted by the least-square algorithm (in other words, all mapped profiles over 50 ms are bounded together rather than being averaged with the same channel before going into the fitting algorithm). The concatenation of the mapped profiles is beneficial for reducing fitting uncertainty when the strike point moves (the coverage and the poloidal spatial resolution of Div-LP array are equivalently improved). In the fitting algorithm, independent variables are optimized in the following range: $\Gamma_{0}\left[\mathrm{Acm}^{-2}\right]\left(j_{s}\right.$ data are used for fitting $) \in[0,1000], \lambda_{j s}[\mathrm{~mm}]$ and $S_{j s}[\mathrm{~mm}] \in[0,100], r_{0}[\mathrm{~mm}] \in[-50,50]$ and $\Gamma_{B G} \in\left[\Gamma_{\min }-\Gamma_{\min , s t d}, \Gamma_{\min }+\Gamma_{\min , s t d}\right]$, where $\Gamma_{\min }$ is the minimum mean value when the concatenated profile is averaged with the same $r$ and $\Gamma_{\text {min,std }}$ is the standard deviation. For H-mode plasmas, only ELMy discharges are used and ELM bursts are removed by referring to the $\mathrm{D}_{\alpha}$ signal during the concatenation process.

\subsection{Data selection and database construction}

In this section, we show the general procedures of the database construction for the $2016 \mathrm{~W}$ divertor campaign (for the 2012 campaign, the procedures are the same). There are 3060 valid discharges with USN configuration $\left(d R_{\text {sep }}(\mathrm{mm}) \in[14,35]\right)$ in the 2016 campaign. This is a 
relatively large number, the preliminary data selection is needed to exclude the discharges for each Div-LP array, where Div-LPs have poor coverage of the divertor $j_{s}$ profile or parallel heat flux $q / /$ profile or there is a large fitting uncertainty for the fits. Specifically, 18 time points are selected evenly for each valid discharge. For each Div-LP array, the measured $j_{s}$ or $q_{/ /}$profiles are mapped to the OMP and concatenated over $50 \mathrm{~ms}$ in the vicinity of each selected time point. Employing fitting method introduced in section 2.2, all 18 concatenated $j_{s}$ or $q_{/ /}$profiles are fitted (for $q / /$ profiles, the same fitting algorithm is used). To enable the fast automatic selection, the concatenated profiles are averaged with the same $r$. And the following parameters are introduced to estimate the quality of the averaged profiles and the fitting uncertainty for the concatenated profiles: $R^{2}, n_{S O L}, r_{S O L-m i n, p e a k}$, and $r_{S O L, t a i l}$. Here, $R^{2}$ is the coefficient of determination evaluated with the averaged profile and the fitted coefficients. $n_{S O L}$ is the number of points in the SOL region of an averaged profile. $r_{S O L-m i n, p e a k}$ is the ratio of the minimum value of an averaged profile in the SOL region to the maximum value of the entire averaged profile. A minimum value of $n_{S O L}$ and a maximum value of $r_{S O L-m i n, p e a k}$ are both required to ensure the good measurement coverage of the divertor $j_{s}$ or $q / /$ profiles in the SOL region. $r_{S O L, \text { tail }}$ is the ratio of the value of the outermost point from the strike point in the SOL region to the minimum value of an averaged profile. A maximum value is needed to minimize the influence of the strike point splitting induced by LHW $[34,35]$. No extra parameter is needed for the PFR region, because the divertor profiles in this region are normally covered and are seldom influenced by LHW. An additional parameter $r_{\text {good, }}$, the ratio of the total number of good averaged profiles to 18 , is used to determine whether to exclude a discharge or not for the data from a Div-LP array.

With the parameters introduced above, we are able to do the preliminary data selection for each Div-LP array, which consists of two steps. For the first step, a relatively loose criteria $\left(R^{2} \geq\right.$ $0.85, n_{S O L} \geq 4, r_{S O L-m i n, p e a k}<0.2, r_{S O L, t a i l}<0.05$ and $\left.r_{\text {good }}>0.5\right)$ is used and the selection results is listed in table 1 . It is clear that only the UI $j_{s}$ data can be used for further analysis, due to the strike point splitting and the poor electron temperature measurements at the upper outer divertor. Then, we focus on analyzing the UI $j_{s}$ data instead of the UO $q_{/ /}$data for the W-divertor operations in this paper. For the second step, a strict criteria $\left(R^{2} \geq 0.9, n_{S O L} \geq 4, r_{S O L-m i n, p e a k}<0.1\right.$, $r_{S O L, \text { tail }}<0.01$ and $\left.r_{\text {good }}>0.8\right)$ is used to select the UI $j_{s}$ data, which makes 217 and 425 discharges available for the UI-D and UI-O Div-LP arrays, respectively.

Table 1. The total number of discharges after the first step of the preliminary data selection of the $j_{s}$ and $q_{/ /}$data for each Div-LP array in the 2016 campaign.

\begin{tabular}{llll}
\hline UI-D & UI-O & UO-D & UO-O
\end{tabular}




\begin{tabular}{lllll}
\hline$j_{s}$ data & 926 & 1481 & 9 & 24 \\
$q / /$ data & 13 & 0 & 0 & 0 \\
\hline
\end{tabular}

After the preliminary data selection, the time range of the different confinement (L-mode or H-mode) phases is manually marked for each discharge. Then, a complete fit of the $j_{s}$ concatenated profiles (over $50 \mathrm{~ms}$ ) is performed for each confinement phase, for each Div-LP array. A secondary data selection is performed by applying $R^{2} \geq 0.95$ and by manually restricting the time range for each individual confinement phase, during which both $\lambda_{j s}$ and $S_{j s}$ have relatively small variations. Figure 2 is an example to demonstrate this selection process. The data points from $3.6 \mathrm{~s}$ to $4.8 \mathrm{~s}$ are removed because $R^{2}<0.95$ and the data points in the shaded area are selected to enter into the database.

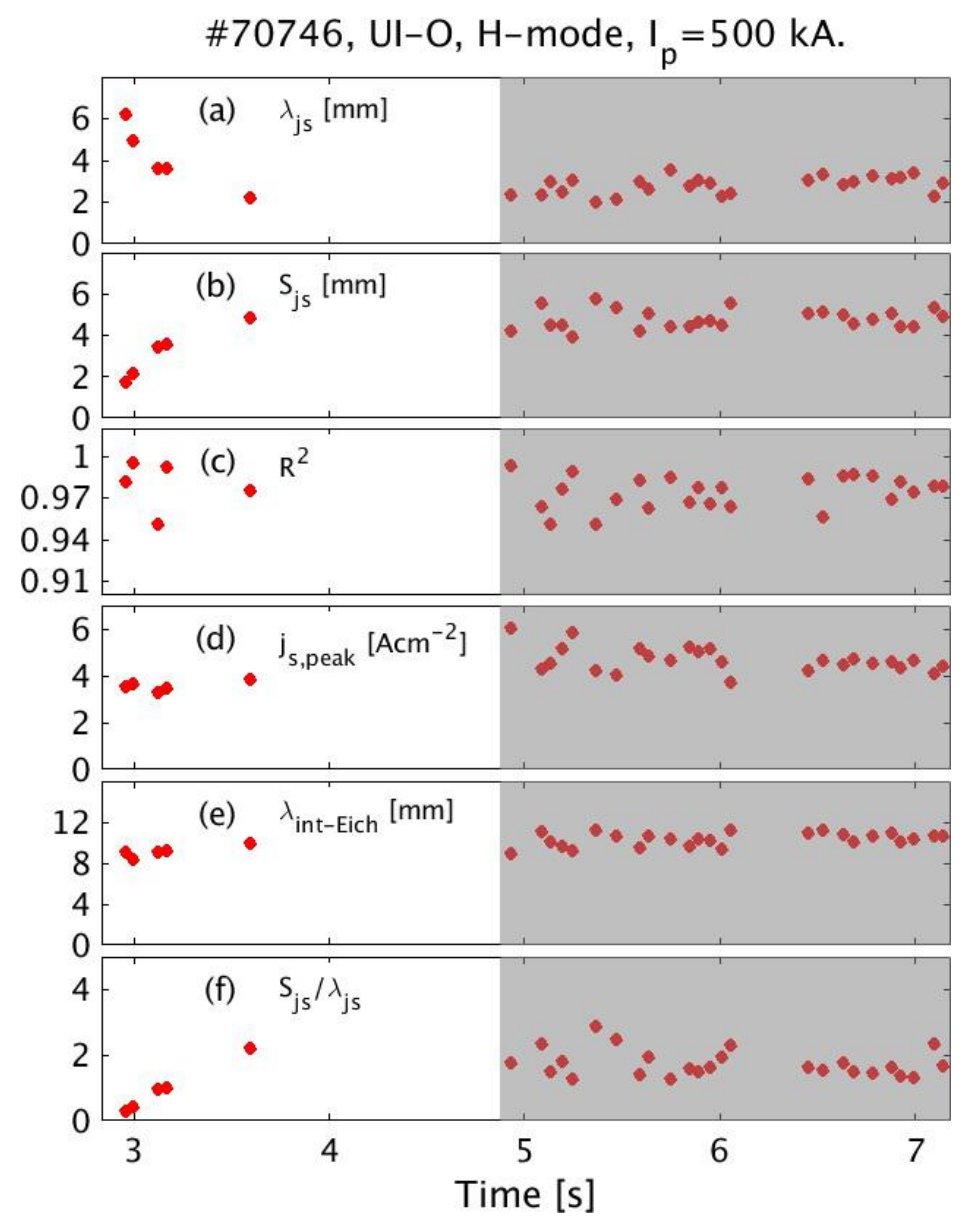

Figure 2. An example of the fitted parameters for the UI-O Div-LP data in an H-mode discharge, where the data points in the shaded area being selected to enter into the database. 
Now we have the fitted parameters $\left(\lambda_{j s}, r_{0}\right.$ and $\left.S_{j s}\right)$ and the characteristic parameters of the averaged profiles $\left(j_{s, p e a k}\right.$ and $\left.\lambda_{\text {int }}\right)$ in our database for each confinement phase, for each Div-LP array. Here, $j_{s, p e a k}$ is the peak value of an averaged profile and $\lambda_{\text {int }}$ is the particle flux integral width of an averaged profile $\left(\lambda_{\text {int }} \equiv \int\left(\Gamma(r)-\Gamma_{B G}\right) d r / \Gamma_{\text {peak }}[5]\right.$, where $\left.\Gamma_{\text {peak }}=j_{s, p e a k} / e\right)$. In addition to these parameters, some other important engineering and physical parameters are also collected into the database: $R, a, B_{t}, I_{p}, B_{p}, \bar{n}_{e}, P_{\text {tot }}, P_{\text {rad }}, \kappa, \delta_{\text {top }}, \delta_{\text {bottom }}, q_{c y l}, W_{M H D}, L_{\text {leg, in }}$ and $L_{\text {leg,out, }}$, where $\bar{n}_{e}$ is the line-averaged electron density, $P_{t o t}$ is the total heating power, $P_{\text {rad }}$ is the total radiated power, $\kappa$ is the plasma elongation, $\delta_{\text {top }}$ and $\delta_{\text {bottom }}$ is the upper and lower triangularity respectively, $q_{c y l}$ is the cylindrical safety factor, $L_{\text {leg, } i n}$ and $L_{\text {leg,out }}$ are the inner and outer divertor leg lengths projected to the poloidal cross section from X-point to the strike point, respectively.

Until now, we finished introducing the procedures of the database construction for the 2016 campaign. For the 2012 campaign, the $\mathrm{UO} j_{s}$ data are available (resulting from low RF heating power in the 2012 campaign), but with relatively poor fitting uncertainty for most of the profiles. In order to keep enough data to investigate the in-out asymmetry of $\lambda_{j s}, R^{2} \geq 0.88$ is applied during the preliminary and secondary data selection processes. Table 2 tabulates the range of the main parameters in the final constructed database. For the data in the 2012 campaign, $\lambda_{j s}$ and $S_{j s}$ have wider windows because of the inclusion of the outer divertor data. Due to the inclusion of Ohmic discharges and the lower heating capability in the 2012 campaign, $P_{t o t}$ and $W_{M H D}$ have narrower windows.

Table 2. The range of the particle flux footprint widths and other main parameters in the constructed database.

\begin{tabular}{llllll}
\hline & $2012(\mathrm{C})$ & $2016(\mathrm{~W})$ & & $2012(\mathrm{C})$ & $2016(\mathrm{~W})$ \\
\hline$\lambda_{j s}[\mathrm{~mm}]$ & $0.89 \sim 18.4$ & $2.03 \sim 6.27$ & $P_{\text {tot }}[\mathrm{MW}]$ & $0.29 \sim 1.59$ & $1.12 \sim 3.40$ \\
$S_{j s}[\mathrm{~mm}]$ & $0.64 \sim 13.4$ & $0.46 \sim 5.99$ & $W_{M H D}[\mathrm{MJ}]$ & $0.01 \sim 0.09$ & $0.05 \sim 0.18$ \\
$B_{t}[\mathrm{~T}]$ & $1.74 \sim 1.84$ & $1.76 \sim 2.44$ & $\delta_{\text {top }}$ & $0.42 \sim 0.50$ & $0.44 \sim 0.55$ \\
$I_{p}[\mathrm{MA}]$ & $0.39 \sim 0.50$ & $0.40 \sim 0.60$ & $\delta_{\text {bottom }}$ & $0.22 \sim 0.32$ & $0.24 \sim 0.30$ \\
$B_{p}[\mathrm{~T}]$ & $0.16 \sim 0.20$ & $0.16 \sim 0.23$ & $\kappa$ & $1.53 \sim 1.73$ & $1.57 \sim 1.67$ \\
$\bar{n}_{e}\left[10^{19} \mathrm{~m}^{-3}\right]$ & $1.53 \sim 5.30$ & $1.98 \sim 4.68$ & $q_{c y l}$ & $2.24 \sim 2.65$ & $2.39 \sim 3.44$ \\
\hline
\end{tabular}

Note: the values are calculated using $96 \%$ data of the distribution close to the mean value. 


\section{$3 \quad$ Results and discussions}

\subsection{Particle flux fall-off width $\lambda_{j s}$}

Using the constructed database, we are able to scale the $\mathrm{W}$-divertor $\lambda_{j s}$ against $B_{p}$ in the 2016 campaign. Figure 3 shows the regression results for both $\mathrm{L}$ - and $\mathrm{H}$-mode plasmas. For interpretation of the results, it should be noted that $\lambda_{j s}$ and $B_{p}$ are averaged with the same discharge to eliminate the inequality of the number of selected profiles for different discharges. The black solid and dashed lines represent the regression results and the regression uncertainties within $95 \%$ confidence interval, respectively. Although the distribution of the data is largely scattered for both L- and H-mode plasmas, both of the scalings generally exhibit a clear decreasing trend with $B_{p}$, which are consistent with previous $\lambda_{j s}$ scalings at the outer $\mathrm{C}$ divertor in EAST $[6,7]$. However, the variation of $B_{p}$ in the database is smaller than previous EAST scalings and the distribution of $B_{p}$ is quite inhomogeneous. This might be one of the reasons for the scattering of the $\lambda_{j s}$ scalings in figure 3, especially for the large uncertainty in the exponents of $B_{p}$. But still it is quite surprising to observe an inverse dependence of $B_{p}$ in the $\lambda_{j s}$ scalings with such small $B_{p}$ variation using a large-number of general $\mathrm{W}$-divertor discharges with varied plasma parameters.
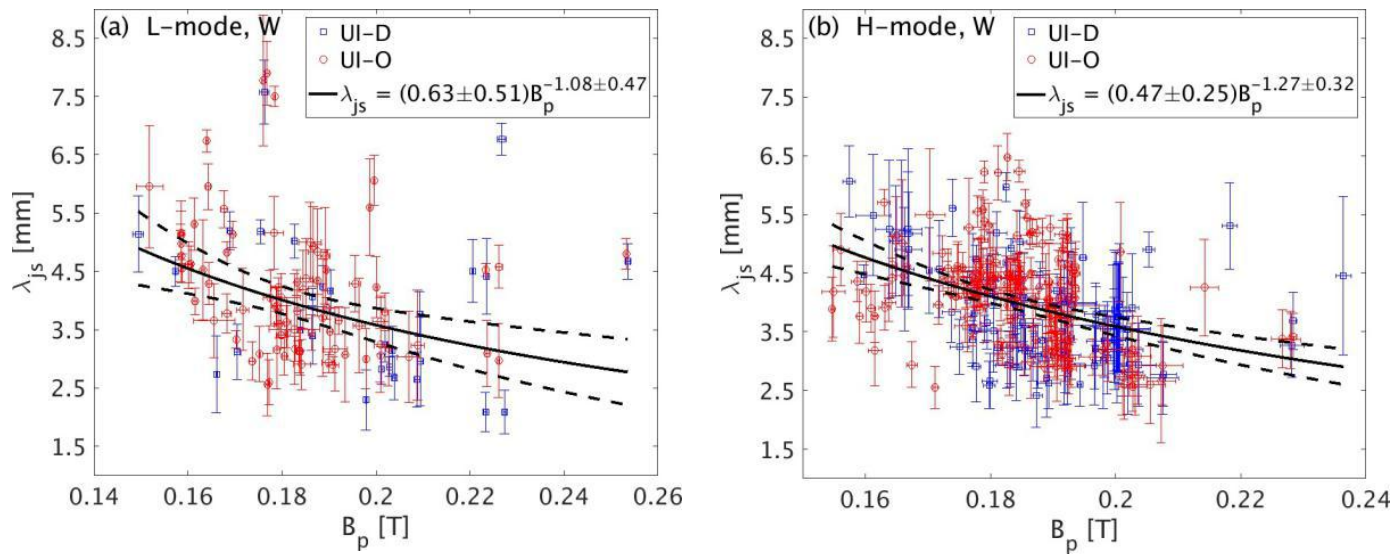

Figure 3. The scaling of $\lambda_{j s}$ against $B_{p}$ for (a) L-mode and (b) H-mode plasmas at the inner W divertor with USN configuration in the 2016 campaign, where the solid and dashed lines are the regression results and the regression uncertainties within $95 \%$ confidential interval, respectively. The blue squares and the red circles are the data from the upper inner Div-LPs located at the port D and port O, respectively.

In figure 3(b), there is no significant difference between the UI-D and UI-O $\lambda_{j s}$ with similar $B_{p}$ for H-mode scaling, since the UI-D and UI-O Div-LPs are toroidally symmetrically 
distributed. However, from the comparison results in figure 4(a) between the UI-D and UI-O $\lambda_{j s}$ without averaging, it is clear that the UI-O $\lambda_{j s}$ is normally larger than the UI-D $\lambda_{j s}$. A more specific comparison of these two averaged profiles of one discharge (EAST\#66683) in figure 4(b) indicates that although the fitted $\lambda_{j s}$ varies a lot between these two profiles, the profiles themselves do not differ much. The possible reasons for this difference could be the sparse distribution, the measurement error, the fluctuations of Div-LPs and/or toroidal asymmetric distribution of particle flux, which make the fitted $\lambda_{j s}$ very sensitive to small profile variations.
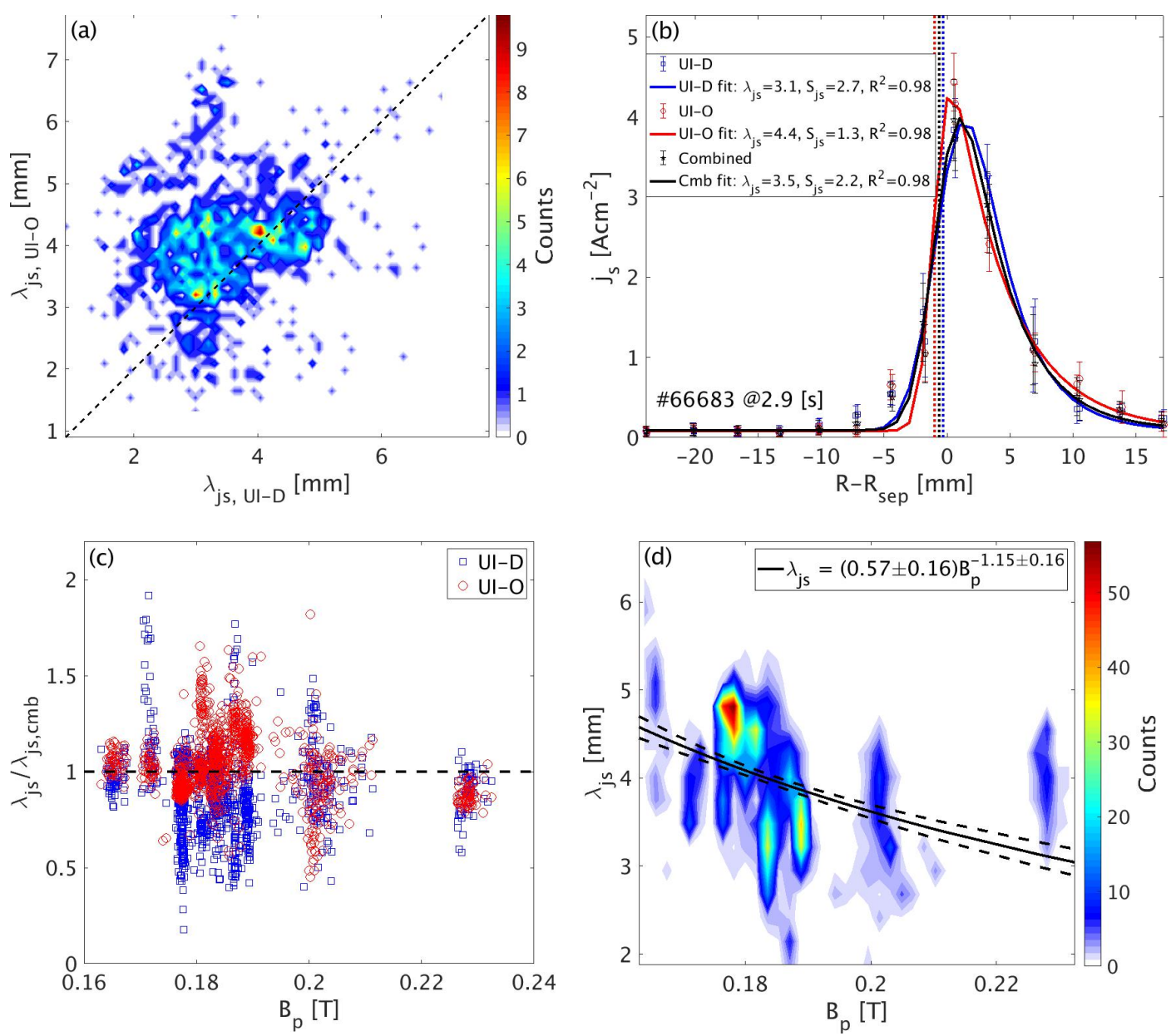

Figure 4. (a) The comparison between the UI-D and UI-O W-divertor $\lambda_{j s}$ in H-mode plasmas. (b) An example of the UI-D and UI-O averaged profiles and the averaged combined profiles as well as their fitting results (solid and dashed lines represent the fitted profiles and the LCFS locations). (c) The comparison among the UI-D, UI-O and combined $\lambda_{j s}$, where the dashed line is the baseline of combined $\lambda_{j s}$. (d) The $\lambda_{j s}$ scaling against $B_{p}$ using the combined database (the solid line and dashed lines represent(s) the regression result and regression uncertainty within $95 \%$ confidential interval, respectively). 
Since we do not know statistically the Div-LP data from which toroidal location are more accurate and considering that the profile difference is relatively small, it is possible to combine the concatenated profiles from these two ports together (forming a combined profile) and to refit again (refer to the black solid line in figure 4(b) for the refitted result). After repeating the combining process for each pair of the UI-D and UI-O profile in the database for H-mode plasmas in the 2016 campaign, we apply $R^{2} \geq 0.95$ to filter out the combined profiles that have relatively large fitting uncertainty (only $5 \%$ profiles are removed). The profile combining process can be treated as an averaging process according to the comparison among the UI-D, UI-O and the combined $\lambda_{j s}$ in figure 4(c). From this figure, we see that the profile combining process reduces the fitting uncertainty by up to $50 \%$. In order to distinguish from the original database in section 2.3, a new further database (combined database) is therefore created based on the newly fitted parameters and the original engineering and physical parameters. We also define a reduced database based on averaging parameters in the combined database by the same heating combination in each discharge to eliminate the inequality of the number of selected profiles. For the probe data in L-mode plasmas, the same approach cannot be employed because there are not enough valid discharges (see figure 3(a)).

Table 3. The median values and the MADs of the important parameters in the combined and reduced (marked with *) databases.

\begin{tabular}{|c|c|c|c|c|c|c|c|}
\hline & Median & $\begin{array}{l}\text { MAD } \\
{[\%]}\end{array}$ & $\begin{array}{l}\text { MAD } \\
{[\%]^{*}}\end{array}$ & & Median & $\begin{array}{l}\text { MAD } \\
{[\%]}\end{array}$ & $\begin{array}{l}\text { MAD } \\
{[\%]^{*}}\end{array}$ \\
\hline$\lambda_{j s}[\mathrm{~mm}]$ & 3.99 & 13.8 & 14.0 & $L_{\text {leg,in }}[\mathrm{m}]$ & 0.23 & 4.16 & 2.70 \\
\hline$S_{j s}[\mathrm{~mm}]$ & 2.68 & 25.1 & 31.0 & $L_{\text {leg,out }}[\mathrm{m}]$ & 0.24 & 2.78 & 1.29 \\
\hline$\lambda_{\text {int }}[\mathrm{mm}]$ & 8.67 & 13.9 & 12.6 & $\delta_{t o p}$ & 0.50 & 2.70 & 1.27 \\
\hline$j_{s, p e a k}\left[\mathrm{Acm}^{-2}\right]$ & 5.40 & 23.9 & 14.5 & $\delta_{\text {bottom }}$ & 0.28 & 1.41 & 1.53 \\
\hline$B_{p}[\mathbf{T}]$ & 0.18 & 2.95 & 5.47 & $\kappa$ & 1.60 & 0.51 & 0.36 \\
\hline$W_{M H D}[\mathrm{MJ}]$ & 0.13 & 13.0 & 18.1 & $R[\mathrm{~m}]$ & 1.91 & 0.21 & 0.16 \\
\hline $\bar{n}_{e}\left[10^{19} \mathrm{~m}^{-3}\right]$ & 3.04 & 7.47 & 4.07 & $a[\mathrm{~m}]$ & 0.46 & 0.53 & 0.60 \\
\hline$P_{t o t}[\mathrm{MW}]$ & 2.33 & 11.4 & 13.5 & $B_{t}[\mathrm{~T}]$ & 2.42 & 0.32 & 0.28 \\
\hline
\end{tabular}

Note: the values are calculated using $96 \%$ data of the distribution close to the mean value. The parameters with relatively large variation $(\mathrm{MAD} \geq 2 \%$ ) are marked in bold and selected for the non-linear regression.

With the combined database, the $\lambda_{j s}$ scaling against $B_{p}$ is refitted in figure $4(\mathrm{~d})$, but with relatively poor fitting quality $\left(R^{2}=0.15\right)$. The vertical structures in this figure implies that the $\lambda_{j s}$ 
scaling may depend on some other parameters, which can be further investigated by employing the multi-variable non-linear regression with the power law function $[4,8]$,

$$
y=C_{0} \prod_{i=1}^{n} x_{i}^{C_{x_{i}}},(i=1,2 \cdots, n)
$$

where $y$ is the particle flux footprint widths, $C_{0}$ and $C_{x_{i}}$ are the coefficients and $x_{i}$ are the independent variables, i.e., the parameters marked in bold in table 3. Since $I_{p}$ and $q_{c y l}$ are correlated with $R, a, B_{p}$ and/or $B_{t}$, they are omitted ( $B_{p}$ is selected because the variation of $I_{p}$ is even smaller according to table 2). $P_{t o t}$ is used to replace the power entering SOL $\left(P_{S O L}=P_{t o t}\right.$ $\left.P_{r a d}\right)$ because of the lack of $P_{r a d}$ measurements in the combined database for a number of discharges. Table 3 lists the median values and the relative median absolute deviations (MADs) of the important parameters in the combined and reduced databases. Since the MADs of $R, a, B_{t}$, $\delta_{\text {bottom }}$ and $\kappa$ are less than $1.5 \%$, they are omitted.

The non-linear regression result using the remaining parameters (marked in bold in table 3) is shown in figure 5(a). Although $R^{2}$ is only 0.48 , it is better than the regression result in figure 4(d). Since the exponents of $\delta_{\text {top }}, L_{\text {leg, in }}$ and $L_{\text {leg,out }}$ in the regression result have larger uncertainties and the variations of these parameters are also smaller (see table 3), they are omitted in the next-step regression. In table 4, we minimize the number of regression parameters gradually. The value of $R^{2}$ in $\# 1$ demonstrates that our ignorance of these three parameters is reasonable. The most interesting issue in $\# 1$ is the positive dependence on $B_{p}$, which is contradictory to the regression result shown in figure 4(d). From the mutual correlations [36] among the regression parameters of \#1 listed in table 5, we learn that there is a strong positive correlation between $B_{p}$ and $W_{M H D}$ (75.4\%). In $\# 2, W_{M H D}$ is removed and the negative dependence on $B_{p}$ is recovered. So the positive dependence on $B_{p}$ in $\# 1$ results from the small variation of $B_{p}$ (see table 3 ) and its strong positive correlation with $W_{M H D}$. Since $R^{2}$ drops from 0.47 to 0.39 when $W_{M H D}$ is removed, we keep $W_{M H D}$ instead of $B_{p}$. In $\# 3, R^{2}$ and the exponents of other parameters do not change much compared with those in $\# 1$, indicating that $W_{M H D}$ is more significant than $B_{p}$ for the $\lambda_{j s}$ scaling. $j_{s, p e a k}$ is removed in $\# 4$ because of its relatively strong negative correlation with $W_{M H D}$ (see table 5). Note that the exponents of $W_{M H D}$ and $\bar{n}_{e}$ have relatively close absolute values, which is similar to the AUG L-mode scaling of $\lambda_{q}$ [8]. Following the same idea that $W_{M H D} / \bar{n}_{e}$ is proportional to $T_{e, e d g e}$ because of the stiffness of pressure profiles [37] in H-mode plasmas [8], \#5 is obtained. Since the correlation of $B_{p}$ to $W_{M H D} / \bar{n}_{e}$ is weaker than that to $W_{M H D}$ (see table 5), $B_{p}$ is added back in $\# 6$, but the exponent is very small and has a very large uncertainty. The same procedure is repeated for the reduced 
database and the results are listed from $\# 7^{*}$ to $\# 12^{*}$. Compared with $\# 4, \# 10^{*}$ has stronger dependences on $W_{M H D}$ and $P_{\text {tot }}$ while weaker dependence on $\bar{n}_{e}$, which makes the replacement to $W_{M H D} / \bar{n}_{e}$ in $\# 11^{*}$ less reasonable. However, from the result shown in $\# 12^{*}$, we see that the dependence on $B_{p}$ is negative and the dependence on $P_{t o t}$ is consistent with that in $\# 6$. The reason for the former observation is that the variation of $B_{p}$ is larger in the reduced database than that in the combined database (see table 3) and the dependence on $W_{M H D} / \bar{n}_{e}$ is also weaker. The reason for the latter one is that $P_{t o t}$ is strongly correlated with $W_{M H D}$ while is weakly correlated with $W_{M H D} / \bar{n}_{e}$ (see table 5). All in all, the $\lambda_{j s}$ scaling is quite dependent on the variations of the regression parameters and how the regression parameters are correlated with each other. Since \#5 has the smallest uncertainties of the regression coefficients and the least number of the regression parameters, we propose it as our scaling of the inner W-divertor $\lambda_{j s}$ for EAST H-mode plasmas (see also figure 5(b)),

$$
\lambda_{j s, U I, H, W}=(0.85 \pm 0.09)\left(W_{M H D} / \bar{n}_{e}\right)^{-0.50 \pm 0.03} P_{t o t}^{-0.06 \pm 0.04} .
$$

The possible reasons for the moderate $R^{2}$ in the above regression might be the measurement errors of the regression parameters and the uncertainty of the magnetic equilibrium reconstruction.
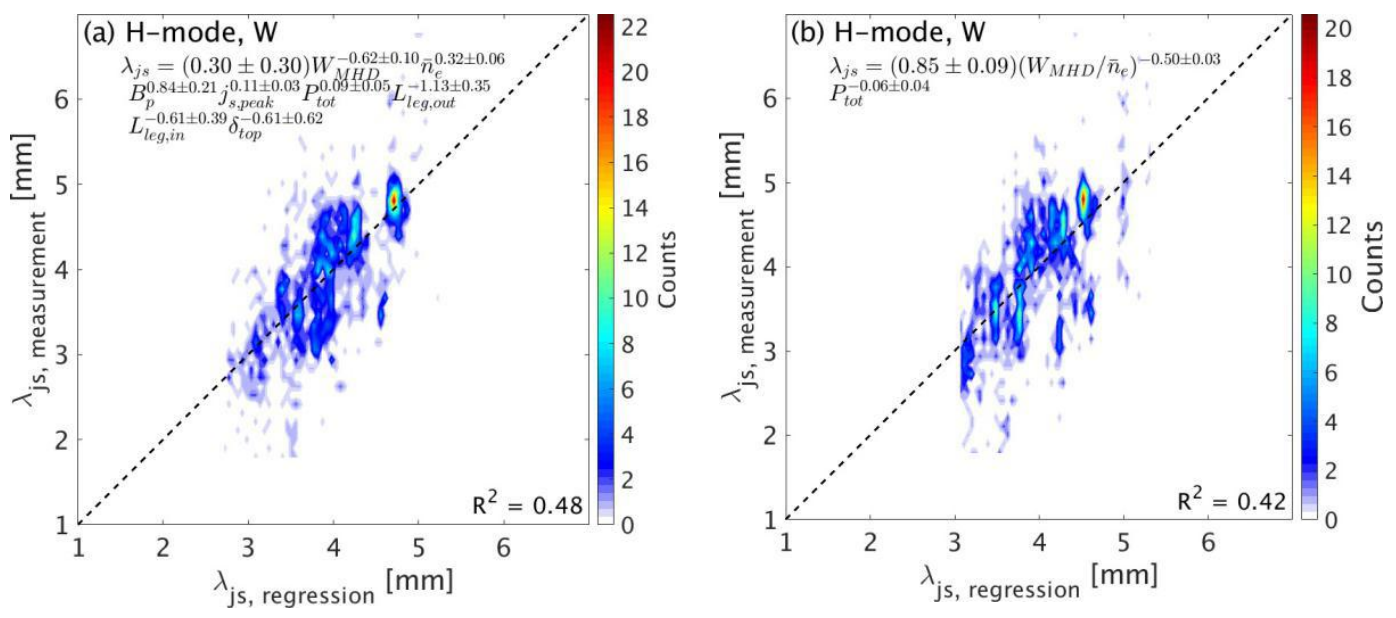

Figure 5. The non-linear regression results of $\lambda_{j s}$ using the combined database: (a) with parameters in table 3 marked in bold and (b) with parameters in the right hand side of Eq. (4).

Table 4. The non-linear regression results of $\lambda_{j s}$ using the combined and reduced (marked with $*$ ) databases. 


\begin{tabular}{lllllllll}
\hline$\#$ & $C_{0}$ & $C_{B_{p}}$ & $C_{W_{M H D}}$ & $C_{\bar{n}_{e}}$ & $C_{W_{M H D} / \bar{n}_{e}}$ & $C_{P_{\text {tot }}}$ & $C_{j_{s, p e a k}}$ & $R^{2}$ \\
\hline 1 & $1.74(0.37)$ & $0.62(0.20)$ & $-0.64(0.09)$ & $0.29(0.05)$ & & $0.07(0.05)$ & $0.10(0.03)$ & 0.47 \\
2 & $1.08(0.24)$ & $-0.44(0.14)$ & & $0.24(0.06)$ & & $-0.13(0.05)$ & $0.21(0.03)$ & 0.39 \\
3 & $1.01(0.13)$ & & $-0.41(0.06)$ & $0.30(0.05)$ & & $-0.02(0.04)$ & $0.11(0.03)$ & 0.46 \\
4 & $0.78(0.09)$ & & $-0.58(0.05)$ & $0.40(0.05)$ & & $-0.01(0.04)$ & & 0.44 \\
5 & $0.85(0.09)$ & & & & $-0.50(0.03)$ & $-0.06(0.04)$ & & 0.42 \\
6 & $0.93(0.18)$ & $0.08(0.14)$ & & & $-0.51(0.04)$ & $-0.05(0.04)$ & & 0.42 \\
$7 *$ & $1.42(1.20)$ & $0.80(0.75)$ & $-0.99(0.32)$ & $0.27(0.17)$ & & $0.19(0.17)$ & $-0.04(0.11)$ & 0.64 \\
$8 *$ & $0.58(0.60)$ & $-0.88(0.63)$ & & $0.16(0.20)$ & & $-0.06(0.19)$ & $0.17(0.11)$ & 0.33 \\
$9 *$ & $0.67(0.31)$ & & $-0.71(0.22)$ & $0.23(0.17)$ & & $0.14(0.17)$ & $-0.01(0.11)$ & 0.61 \\
$10 *$ & $0.68(0.26)$ & & $-0.70(0.14)$ & $0.23(0.16)$ & & $0.13(0.16)$ & & 0.62 \\
$11 *$ & $0.94(0.40)$ & & & & $-0.46(0.13)$ & $-0.04(0.16)$ & & 0.49 \\
$12 *$ & $0.54(0.46)$ & $-0.47(0.61)$ & & & $-0.39(0.16)$ & $-0.02(0.16)$ & & 0.51 \\
\hline
\end{tabular}

Table 5. The mutual correlations among the selected parameters in the combined and reduced (marked with *) databases.

\begin{tabular}{|c|c|c|c|c|c|c|}
\hline$W_{M H D}$ & $\bar{n}_{e}$ & $W_{M H D} / \bar{n}_{e}$ & $P_{t o t}$ & $\delta_{t o p}$ & $j_{s, p e a k}$ & Corr. [\%] \\
\hline 75.4 & 0.40 & 50.6 & -11.4 & 18.3 & -40.7 & $B_{p}$ \\
\hline \multirow[t]{11}{*}{$84.0^{*}$} & $13.4^{*}$ & $55.3^{*}$ & $22.5^{*}$ & $-0.77 *$ & $-47.11 *$ & \\
\hline & 2.80 & 74.5 & 25.7 & 14.0 & -57.4 & $W_{M H D}$ \\
\hline & $18.4^{*}$ & 69.2* & $45.7^{*}$ & $-10.4^{*}$ & $-62.2 *$ & \\
\hline & & -62.1 & 26.4 & -42.2 & 38.2 & $\bar{n}$ \\
\hline & & $-56.9^{*}$ & $30.7^{*}$ & $-31.1^{*}$ & $16.8^{*}$ & \\
\hline & & & 9.09 & 29.3 & -68.8 & $W_{M H D} / \bar{n}$ \\
\hline & & & $16.7^{*}$ & $7.52^{*}$ & $-64.3^{*}$ & \\
\hline & & & & -34.5 & 3.66 & $P_{t o t}$ \\
\hline & & & & $-37.2 *$ & $-0.40^{*}$ & \\
\hline & & & & & -48.5 & $\delta_{\text {top }}$ \\
\hline & & & & & $-36.8^{*}$ & \\
\hline
\end{tabular}

Note: the values larger than $45 \%$ are marked in bold.

\subsection{Particle flux spreading width $S_{j s}$}

For the scaling of the particle flux spreading width in the PFR, $S_{j s}$, we use the combined database and start with the parameters marked in bold in table 3. The regression result is shown in figure 6(a). After omitting $L_{\text {leg, in }}$ and $L_{\text {leg,out }}$ (the uncertainty of their exponents are large), the number of the regression parameters are minimized in table 6 . From $\# 2$ to $\# 3, j_{s, p e a k}$ and $B_{p}$ are 
removed step by step because of their strong correlations with $W_{M H D}$ and/or $\delta_{\text {top }}$ (see table 5). During the process of the reduction of the regression parameter number for $S_{j s}$, the exponents of $W_{M H D}, \bar{n}_{e}$ and $P_{t o t}$ change significantly compared with that for $\lambda_{j s}$. This means that $S_{j s}$ does not depend on these three parameters significantly in the combined database. To get a more robust scaling, the combined database should be extended to include more data. So, we propose \#3 as the scaling of the inner W-divertor $S_{j s}$ for EAST H-mode plasmas (see also figure 6(b)),

$$
S_{j s, U I, H, W}=(1.44 \pm 0.36) W_{M H D}^{0.93 \pm 0.09} \bar{n}_{e}^{0.25 \pm 0.09} P_{t o t}^{0.07 \pm 0.09} \delta_{t o p}^{-3.16 \pm 0.38} .
$$

Compared with the $\lambda_{j s}$ scaling in Eq. (4), the $S_{j s}$ scaling has an extra strong negative dependence on $\delta_{\text {top }}$, shows a similar dependence on $P_{t o t}$ and has a weaker positive dependence on $\bar{n}_{e}$, but exhibits a positive dependence on $W_{M H D}$.
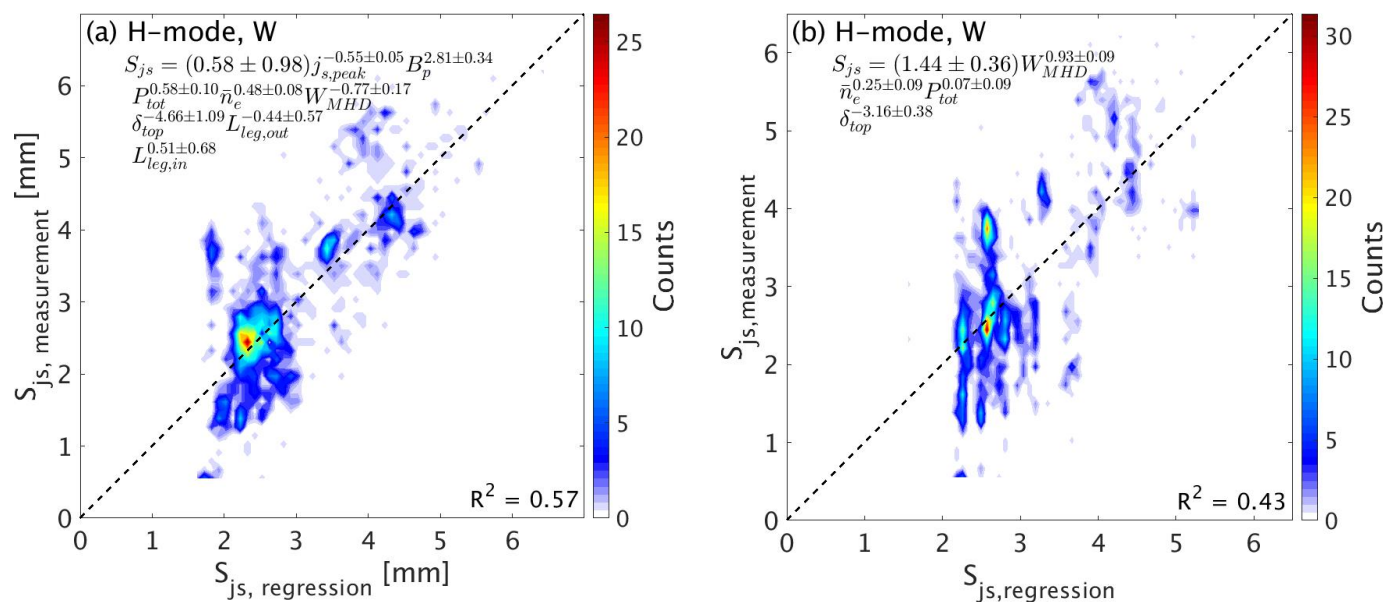

Figure 6. The non-linear regression results of $S_{j s}$ using the combined database: (a) with parameters in table 3 marked in bold and (b) with parameters in the right hand side of Eq. (5).

Table 6. The non-linear regression results of $S_{j s}$ using the combined database.

\begin{tabular}{lllllllll}
\hline$\#$ & $C_{0}$ & $C_{B_{p}}$ & $C_{W_{\text {MHD }}}$ & $C_{\bar{n}_{e}}$ & $C_{P_{\text {tot }}}$ & $C_{\delta_{\text {top }}}$ & $C_{j_{s, p e a k}}$ & $R^{2}$ \\
\hline 1 & $2.31(0.75)$ & $2.70(0.31)$ & $-0.76(0.16)$ & $0.47(0.08)$ & $0.58(0.10)$ & $-4.47(0.35)$ & $-0.54(0.05)$ & 0.57 \\
2 & $6.06(2.25)$ & $1.91(0.34)$ & $0.27(0.16)$ & $0.16(0.09)$ & $0.37(0.11)$ & $-3.59(0.36)$ & & 0.46 \\
3 & $1.44(0.36)$ & & $0.93(0.09)$ & $0.25(0.09)$ & $0.07(0.09)$ & $-3.16(0.38)$ & & 0.43 \\
\hline
\end{tabular}

\subsection{Comparison with the scalings of the heat flux footprint widths}


In principle, the heat flux fall-off width can be obtained by fitting the measured divertor heat flux profile, which does not work in this paper due to the poor electron temperature measurements (see section 2.3). However, the heat flux fall-off width $\lambda_{q}$ is approximately equal to the particle flux fall-off width $\lambda_{j s}$ for the Div-LP measurements according to previous studies $[6,7,38,39]$. The parallel heat flux $q_{/ /}$measured by Div-LPs is usually evaluated with, $q_{/ /}=\gamma j_{s} T_{e} / e$, where $\gamma$ is the sheath transmission factor [6,38]. In EAST [6,7] and MAST [39], $\lambda_{q} \approx \lambda_{j s}$ is found to be satisfied in both L- and H-mode plasmas, indicating that the electron temperature fall-off width is much larger than the electron density fall-off width at the divertor target. Moreover, the measured electron/ion temperature profile at the divertor target is observed to be flattened in AUG [40], C-Mod [41], EAST [42] and DIII-D [43]. Figure 7 shows the electron temperature profiles measured by Div-LPs in L- and H-mode plasmas. Although the measurement error is relatively large, we can still see that the profile is broad or flat, which means that it is appropriate to make the approximation, $\lambda_{q} \approx \lambda_{j s}$. The flatness of the temperature profile at the divertor target suggests that the divertor heat flux is dominated by the heat convection. This is especially understandable for the H-mode plasmas, because the electron-ion collision is too weak to satisfy the Spitzer-Härm conduction, leading to a reduction of the parallel conduction. This picture is supported by experimental result in C-Mod H-mode plasmas [41], where the divertor heat flux is better recovered from the profiles at the OMP through the simple pressure map (similar to the flux-limited conduction) instead of the Spitzer-Härm conduction.
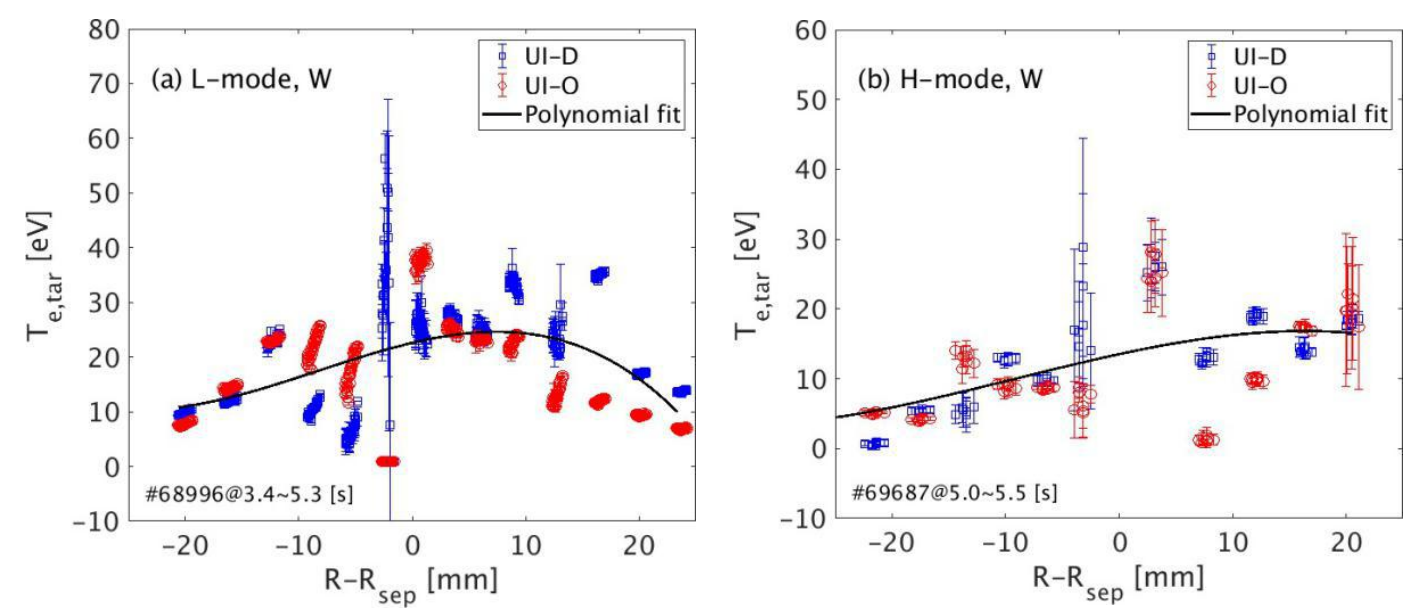

Figure 7. The measured electron temperature profiles by Div-LPs for (a) L-mode (b) Hmode plasmas. The blue squares and the red circles are the data from the upper inner DivLPs located at the port $\mathrm{D}$ and port $\mathrm{O}$, respectively. 
With the approximation discussed above, the $\lambda_{j s}$ scaling in Eq. (4) can be compared with the $\lambda_{q}$ scaling in previous studies. The weak scaling dependence on the heating power is consistent with many studies $[5,8,13,39]$. The scaling dependence on $W_{M H D} / \bar{n}_{e}$ is surprisingly consistent with recent C-Mod $\lambda_{q}$ scaling in L- I- and H-mode plasmas, $\lambda_{q}=0.91 \bar{p}^{-0.48}$ [44], where $\bar{p}$ is the volume-averaged core plasma pressure and is proportional to $W_{M H D}$. For stiff pressure profiles, $W_{M H D} / \bar{n}_{e} \propto T_{e, e d g e}$, then Eq. (4) means $\lambda_{j s, U I, H, W} \propto T_{e, e d g e}^{-0.50}$. Compared with the AUG L-mode scaling, $\lambda_{q} \propto T_{e, e d g e}^{-0.93}$ [8], the scaling dependence on $T_{e, e d g e}$ is weaker for the $\lambda_{j s}$ scaling. Since the electron temperature in the SOL is lower in L-mode plasmas, we may expect that the electron Spitzer-Härm heat conduction dominates the parallel heat flux in L-mode plasmas while the electron and ion heat convection and the reduced electron heat conduction (a combination of the Spitzer-Härm and the flux-limited heat conduction) dominate the parallel heat flux in H-mode plasmas. The Spitzer-Härm heat conduction ( $\left.\propto T_{e}^{7 / 2}\right)$, the flux-limited heat conduction $\left(\propto T_{e}^{3 / 2}\right)$ and the heat convection $\left(\propto T_{e}^{3 / 2}\right.$ ) have different temperature dependences, which could explain the different scaling dependence on $T_{e, e d g e}$ between the H-mode $\lambda_{j s}$ scaling and the AUG L-mode $\lambda_{q}$ scaling. In addition, the weaker temperature dependence for the $\lambda_{j s}$ scaling also confirms that it is appropriate to approximate $\lambda_{q}$ by $\lambda_{j s}$ in H-mode plasmas. The negative scaling dependence on $T_{e, e d g e}$ in this paper is different from that in the heuristic driftbased model that has positive scaling dependence $\left(\lambda_{\text {SOL }} \propto T_{\text {sep }} /\left(B_{p} c_{s}\right)\right.$ [13], where $T_{\text {sep }}$ is the electron/ion temperature at the separatrix and $c_{s}$ is the ion sound speed) and is consistent with the numerical scaling in L-mode plasmas by the HESEL code $\left(\lambda_{q} \propto T_{e, L C F S}^{-0.44}[21]\right.$, where $T_{e, L C F S}$ is the electron temperature at the LCFS). Despite of the different temperature dependence, the heuristic drift-based model predicts results consistent with the Eich scaling, which might imply that the scaling dependence on the edge electron temperature is not as significant as the poloidal magnetic field (or the plasma current).

As for the $S_{j s}$ scaling in Eq. (5), the positive dependence on $W_{M H D}$ is favorable because $S_{j s}$ will dominate the plasma wetted area at the divertor targets in high $W_{M H D}$ scenarios where $\lambda_{j s}$ is supposed to be small according the $\lambda_{j s}$ scaling in Eq. (4). However, this positive dependence is different from previous studies $[8,11,45,46]$, where the heat flux spreading width $S_{q}$ has a negative dependence on $B_{p}$ or $I_{p}$, meaning that $S_{q}$ has a negative dependence on $W_{M H D}$ in our database (normally there is a strong positive correlation between $B_{p}$ and $W_{M H D}$ ). Since $W_{M H D}$ is not significant in the $S_{j s}$ scaling, the combined database needs to be extended to confirm this positive dependence in future studies. 


\subsection{Comparison of $\mathbf{W}$-divertor $\lambda_{j s}$ with $\mathrm{C}$-divertor $\lambda_{j s}$}

The previous EAST $\lambda_{j s}$ scalings (obtained by Div-LPs embedded in the outer C divertor) are [6, 7]

$$
\begin{aligned}
& \lambda_{E A S T, L, C}=1.34 B_{p}^{-1.21}, \\
& \lambda_{E A S T, H, C}=1.53 B_{p}^{-1.26} .
\end{aligned}
$$

Note that the $\lambda_{q}$ scaling is used because there is no $\lambda_{j s}$ scaling against $B_{p}$ for H-mode plasmas in Ref. [6]. This replacement is reasonable according to the discussion in section 3.3. The scaling dependences on $B_{p}$ in Eq. (6) is quite consistent with the Eich $\lambda_{q}$ scaling (obtained by IR camera at the outer divertor) [5],

$$
\lambda_{\text {Eich }}=(0.63 \pm 0.08) B_{p}^{-1.19 \pm 0.08},
$$

but with about two times larger amplitude. The similar scaling dependence on $B_{p}$ between the particle flux and heat flux fall-off widths might result from either the heat convection dominating the SOL parallel heat flux. Or the scaling dependence on $B_{p}$ is actually the scaling dependence on the parallel connection length $L_{/ /}$with the following approximations, $\lambda_{j s} \approx$ $L /\left(D_{/ / /} D_{\perp}\right)^{1 / 2}$ and $\lambda_{q} \approx L /\left(\chi_{/ /} \chi_{\perp}\right)^{1 / 2}$, where $D_{/ /}$and $D_{\perp}$ are the parallel and perpendicular particle diffusivities and $\chi_{/ /}$and $\chi_{\perp}$ are the parallel and perpendicular heat conductivities, and we use the

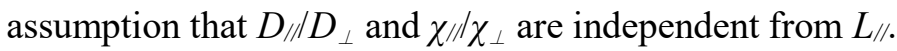

According to section 3.1, the $\lambda_{j s}$ scalings against $B_{p}$ (obtained by Div-LPs at the upper inner $\mathrm{W}$ divertor in the 2016 campaign) can be summarized as,

$$
\begin{aligned}
& \lambda_{j s, U I, L, W}=(0.63 \pm 0.51) B_{p}^{-1.08 \pm 0.47}, \\
& \lambda_{j s, U I, H, W}=(0.47 \pm 0.25) B_{p}^{-1.27 \pm 0.32} .
\end{aligned}
$$

In order to compare the $\mathrm{W}$-divertor $\lambda_{j s}$ with the $\mathrm{C}$-divertor $\lambda_{j s}$, the inner $\mathrm{C}$-divertor data in the 2012 campaign have also been analyzed. However, due to the small variation of $B_{p}$ for the Cdivertor data, a scaling of $\lambda_{j s}$ is not possible. Since the scaling dependence on $B_{p}$ for $\lambda_{j s}$ and $\lambda_{q}$ is robust, we assume that a similar scaling dependence also applies to the C-divertor $\lambda_{j s}$. By normalizing $\lambda_{j s}$ with respect to Eq. (7), the comparison results are shown in figure 8. Note that all the data are averaged with the same discharge. The black and green solid lines represent Eq. (7) and Eq. (6), respectively. The dashed lines represent the mean value of the normalized $\lambda_{j s}$ for the data points with the same color. 
From figure 8, we immediately learn that there is no significant difference between the inner $\mathrm{W}$-divertor $\lambda_{j s}$ and the inner $\mathrm{C}$-divertor $\lambda_{j s}$ in both $\mathrm{L}$ - and $\mathrm{H}$-mode plasmas. This means that $\lambda_{j s}$ is not significantly influenced by the change of divertor material, which is consistent with the result that $\lambda_{q}$ does not differ much between $\mathrm{C}$ and $\mathrm{W}$ divertors in AUG and JET H-mode plasmas [47]. Remember that the direction of $B_{t}$ is configured oppositely between the 2016 and 2012 campaigns, indicating that statistically the direction of $B_{t}$ does not influence $\lambda_{j s}$ significantly. This is beyond our expectation, since the directions of the Pfirsch-Schlüter flows, the $\mathrm{B} \times \nabla \mathrm{B}$-drift and the ExB-drift change when $B_{t}$ is reversed [48]. Thus the particle flux at the inner divertor is largely influenced by the direction of $B_{t}[49]$. There are few studies on the influence of the direction of $B_{t}$ on the particle flux width. The dedicated experiments in TCV [9] and AUG [23] also show that the heat flux width at the inner divertor does not change significantly when $B_{t}$ is reversed. A possible explanation could be that the reversal of $B_{t}$ changes only the amplitude, not the shape of the particle flux profile at the inner divertor.

The comparison of $\lambda_{j s}$ between L- and H-mode plasmas for the $\mathrm{W}$ divertor reveals that there is nearly no difference between them $\left(\lambda_{j s, U I, L, W} / \lambda_{\text {Eich }}=0.87 \pm 0.26\right.$ and $\lambda_{j s, U I, H, W} / \lambda_{\text {Eich }}=0.85 \pm$ 0.16). As for the $\mathrm{C}$ divertor, $\lambda_{j s}$ in L-mode plasmas is about $25 \%$ larger $\left(\lambda_{j s, U, L, C} / \lambda_{j s, U, H, C}=1.24 \pm\right.$ $0.48)$ and $25 \%$ smaller $\left(\lambda_{j s, U O, L, C} / \lambda_{j s, U O, H, C}=0.75 \pm 0.16\right)$ than that in $H$-mode plasmas at the inner and outer divertors, respectively. Considering that the C-divertor data are not as good as the Wdivertor data, $\lambda_{j s}$ in our database seems to have no significant difference between L- and H-mode plasmas. Compared with L-mode plasmas, the plasma temperature is higher for H-mode plasmas, which leads to smaller $\lambda_{j s}$ if the negative scaling dependence on the plasma temperature in $\mathrm{H}$-mode plasmas can also be applied to the L-mode plasmas. The gradients of plasma pressure and temperature are also larger for H-mode plasmas, which enhances the $\mathrm{B} \times$ DB-drift, the poloidal E $\times$ B-drift and the Pfirsch-Schlüter flow. For the 2016 campaign data (USN, forward field), the ion $\mathrm{B} \times \nabla \mathrm{B}$-drift points downwards, the poloidal $\mathrm{E} \times \mathrm{B}$-drift points from the outer divertor to the inner divertor in the SOL and the Pfirsch-Schlüter flow points upwards towards the outer divertor at the Low field side. According to previous studies in C-Mod [48] and EAST [49], the Pfirsch-Schlüter flow dominates the parallel flow at the low field side, where most of the particles are expelled into the SOL from the main plasma. Then the inner W-divertor $\lambda_{j s}$ is probably smaller in H-mode plasmas where the PfirschSchlüter flow is stronger. The radial turbulent transport is weaker in H-mode plasmas, which also make $\lambda_{j s}$ smaller. With these differences in mind, we expect that the inner W-divertor $\lambda_{j s}$ should be smaller in H-mode plasmas, which does not agree with the statistical results shown in figure 8. Since $\lambda_{q}$ is smaller in H-mode plasmas with NBI heating scheme in EAST [11] and 
AUG [8] and $\lambda_{q}$ is similar in L- and H-mode plasmas with RF heating scheme [11], our databases should include the data in the NBI-heated plasmas to confirm that the RF heating scheme mitigates the difference between the L-mode $\lambda_{j s}$ and the H-mode $\lambda_{j s}$ in future study.
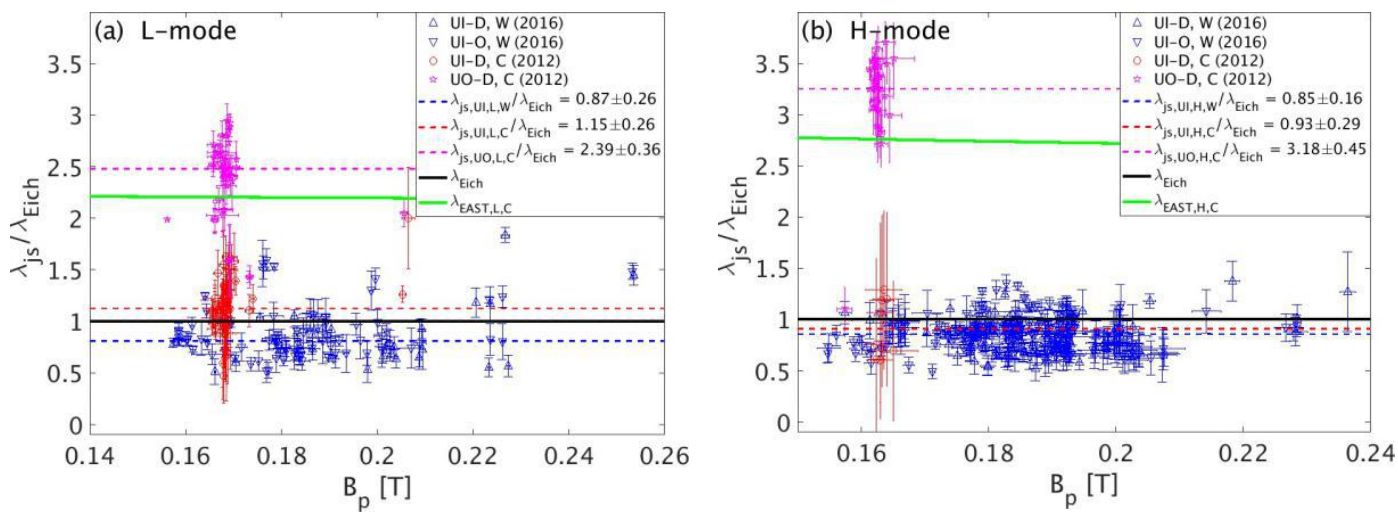

Figure 8. The comparisons of $\lambda_{j s}$ between the $\mathrm{W}$ and $\mathrm{C}$ divertors in EAST for (a) L-mode and (b) H-mode plasmas.

\subsection{In-out asymmetry of C-divertor $\lambda_{j s}$}

Since the outer C-divertor $\lambda_{j s}$ is also included in figure 8, we can compare the inner and outer $\lambda_{j s}$. It is clear that the outer C-divertor $\lambda_{j s}$ agrees with previous EAST $\lambda_{j s}$ scalings in both Land $\mathrm{H}$-mode plasmas and there is a clear in-out asymmetry for the $\mathrm{C}$-divertor $\lambda_{j s}$. For $\mathrm{C}$-divertor $\lambda_{j s}$ in the 2012 campaign (reversed field), the ion B $\times \nabla B$-drift points upwards, the poloidal $\mathrm{E} \times$ B-drift points from the inner divertor to the outer divertor in the SOL and oppositely in the PFR, and the Pfirsch-Schlüter flow is directed downwards at the low field side away from the outer divertor. Thus we see that the particle flux at the inner divertor is stronger than that at the outer divertor in figure 9, where the in-out averaged profiles are inserted in L-mode and Ohmic plasmas. The in-out asymmetry of $\lambda_{j s}$ is quantitatively explained to be geometry related with DN [13] and USN [23] configurations in the heuristic drift-based model. Adjusted to the C-divertor data, it writes,

$$
\lambda_{j s, U O} / \lambda_{j s, U I}=\left(1+\delta_{t o p}\right) /\left(1-\delta_{t o p}\right)
$$

The plot of $\lambda_{j s, o u t} / \lambda_{j s, \text { in }}$ against $\left(1+\delta_{t o p}\right) /\left(1-\delta_{t o p}\right)$ in L-mode plasmas is shown in figure 9 (a). Obviously, this asymmetry does not fit very well with the heuristic drift-based model. Averagely, $\lambda_{j s, \text { out }} / \lambda_{j s, \text { in }} \approx 1.82$ and $\left(1+\delta_{\text {top }}\right) /\left(1-\delta_{\text {top }}\right) \approx 2.60$, i.e., in a narrow window. As mentioned above, the RF heating normally influences $\lambda_{j s}$, which may also contribute to the in-out asymmetry. However, the comparison of the in-out $\lambda_{j s}$ using Ohmic discharges in our database 
in figure 9 (b) shows similar result to that in figure 9 (a) $\left(\lambda_{j s, \text { out }} / \lambda_{j s, \text { in }} \approx 1.69\right)$. In AUG L-mode plasmas, the in-out asymmetry of $\lambda_{q}$ is found to be related to the edge electron density in the plasma density ramping up experiments [8]. Their explanation for this phenomena is that the lower inner divertor is more closed than the outer divertor in AUG, which leads to different inout parallel velocity. Since the upper divertor of EAST in the 2012 campaign was not closed (see the lower divertor in figure 1(b), which was symmetric to the upper divertor in 2012), the in-out asymmetry of the parallel velocity could results from the poloidal ExB flow, the PfirschSchlüter flow and/or the parallel flow due to the ballooning asymmetry [48]. We recall that the inner $\lambda_{j s}$ does not change significantly when the toroidal magnetic field is reversed (see section 3.4). This might indicate that the ballooning asymmetry plays an important role in the in-out asymmetry of $\lambda_{j s}$. Considering that the C-divertor data are not as good as that in the combined database for the $\mathrm{W}$ divertor and there is no data for the outer $\mathrm{W}$ divertor in forward field, more discharges have to be analyzed to further investigate this in-out asymmetry.
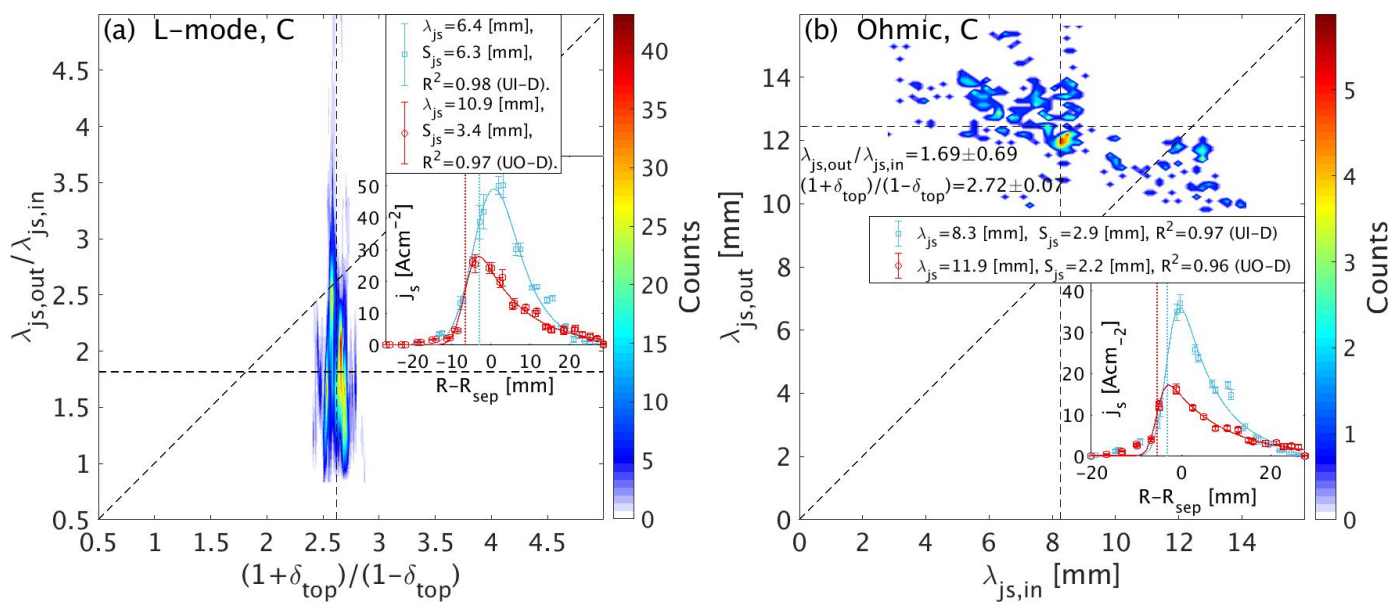

Figure 9. (a) The dependence of $\lambda_{j s, \text { out }} / \lambda_{j, \text { in }}$ on top triangularity (the horizontal and vertical dashed lines represent the median values) and an example of the in-out $j_{s}$ averaged profiles in L-mode plasmas. (b) The in-out asymmetry of $\lambda_{j s}$ and an example of the in-out $j_{s}$ averaged profiles in Ohmic plasmas.

\section{Summary}

In this paper, the upper $\mathrm{W}$-divertor particle flux footprint widths $\left(\lambda_{j s}\right.$ and $\left.S_{j s}\right)$ have been statistically analyzed by using a large-number of Div-LP data in EAST L- and H-mode plasmas. This is the first time to characterize the particle flux footprint for the upper divertor after its upgrade into W structure in 2014. The Div-LP data from the 2016 campaign (W divertor) are 
chosen for detailed analysis and the data from the 2012 campaign ( $\mathrm{C}$ divertor) are used for comparisons. A detailed procedure of the data selection and the database construction is provided. Several parameters characterizing measured Div-LP profiles and their fitting quality are introduced for the automatic preliminary data selections. Further, a database has been constructed by collecting the additional engineering and physical parameters. Taking advantage of the toroidally distributed Div-LP arrays at the upper W divertor, a combined database for Hmode plasmas in the 2016 campaign has been created by fitting the measured particle flux profiles from port $\mathrm{D}$ and port $\mathrm{O}$ (toroidally separated by $112.5^{\circ}$ ) together. It is shown that the fitting uncertainty for $\lambda_{j s}$ is reduced by up to $50 \%$.

Using the constructed database, scaling of the inner $\mathrm{W}$-divertor $\lambda_{j s}$ is performed for both $\mathrm{L}$ and H-mode plasmas. It is found that the scaling dependences on $B_{p}$ is very consistent with previous $\lambda_{j s}$ scalings at the outer $\mathrm{C}$ divertor in EAST, despite the rather small variation of $B_{p}$ in the database. The non-linear regressions of $\lambda_{j s}$ and $S_{j s}$ have been carried out based on the combined database at the inner $\mathrm{W}$ divertor in $\mathrm{H}$-mode plasmas. The non-linear regression result of $\lambda_{j s}$ shows $\lambda_{j s} \propto\left(W_{M H D} / \bar{n}_{e}\right)^{-1 / 2}$ and a very weak dependence on $P_{t o t}$. With the approximation, $\lambda_{q}$ $\approx \lambda_{j s}$, for H-mode plasmas, the $\lambda_{j s}$ scaling is compared with previous $\lambda_{q}$ scalings. It is found that the exponent of $W_{M H D} / \bar{n}_{e}$ is about half of that in the AUG L-mode scaling [8] (could be explained by the different temperature dependences for the parallel conduction between L- and $\mathrm{H}$-mode plasmas), and agrees quite well with the exponent of the volume-averaged plasma core pressure in the C-Mod L- I- and H-mode scaling [38]. For the non-linear regression result of $S_{j s}$, there is/are a strong positive dependence on $W_{M H D}$, weak dependences on $\bar{n}_{e}$ and $P_{\text {tot }}$ and a very strong negative dependence on $\delta_{\text {top }}$.

With the C-divertor data in the 2012 campaign, the comparison of $\lambda_{j s}$ between the inner $\mathrm{W}$ and $\mathrm{C}$ divertors has been made. It appears that statistically neither the change of divertor material nor the direction of toroidal magnetic field has significant influence on $\lambda_{j s}$. The comparison of $\lambda_{j s}$ between L- and H-mode plasmas shows no significant difference, which might be resulting from the RF heating scheme in EAST [11]. The in-out asymmetry of $\lambda_{j s}$ has been observed and can not be fully explained by the heuristic drift-based model and/or the RF heating scheme.

Since in the 2016 campaign EAST was operated at a rather small range of $I_{p}$, the variation of $B_{p}$ is quite small in our database. This leads to the positive dependences on $B_{p}$ (negative dependence is recovered in the $\lambda_{j s}$ scaling, if the reduced database is employed) and the large regression uncertainties for the $\lambda_{j s}$ and $S_{j s}$ scalings. Because the LHW heating that leads to the 
strike point splitting is normally employed in EAST, there are no data for the outer $\mathrm{W}$ divertor in our database to scale $\lambda_{j s}$ and $S_{j s}$ and to study the in-out asymmetry of the W-divertor $\lambda_{j s}$. In EAST, the wall is normally coated by lithium, which might influence the in-out asymmetry of $\lambda_{j s}$. All of these issues shall be further investigated by extending the databases in future work.

\section{Acknowledgments}

This work was supported by National Key Research and Development Program (Nos. 2016YFA0400600, 2017YFA0402500, 2017YFE0301300), China Scholarship Council (No. 201704910761), National Natural Science Foundation of China (No. 11575236) and National Magnetic Confinement Fusion Science Program of China (Nos. 2015GB101000, 2015GB103000). This work was also partly supported by AHNFS (No. 1808085J07), Users with Excellence Project of Hefei Science Center CAS (No.2018HSC-UE008), Key Research Program of Frontier Sciences (CAS, No. QYZDB-SSW-SLH001), K. C. Wong Education Foundation, US Dept. of Energy Contracts DE-AC02-09CH11466.

\section{References}

[1] Loarte A et al 2007 Nucl. Fusion 47 S203-S263

[2] Loarte A et al $1999 \mathrm{~J}$. Nucl. Mater. 266-269 587-592

[3] Eich T et al 2011 Phys. Rev. Lett. 107215001

[4] Makowski M A et al 2012 Phys. Plasmas 19056122

[5] Eich T et al 2013 Nucl. Fusion 53093031

[6] Wang L et al 2014 Nucl. Fusion 54114002

[7] Liu J B et al 2015 Fusion Engineering and Design 100 301-306

[8] Sieglin B et al 2016 Plasma Phys. Control. Fusion 58055015

[9] Maurizio R et al 2018 Nucl. Fusion 58016052

[10] Wang L et al 2017 Nuclear Materials and Energy 12 221-226

[11] Deng G Z et al 2018 Plasma Phys. Control. Fusion 60045001

[12] Faitsch M et al 2018 Plasma Phys. Control. Fusion 60045010

[13] Goldston R J 2012 Nucl. Fusion 52013009

[14] Counsell G F et al 1999 J. Nucl. Mater. 266-269 91-98

[15] Stangeby P C et al 2010 Nucl. Fusion 50125003

[16] Meier E T et al 2016 Plasma Phys. Control. Fusion 58125012

[17] Deng G Z et al 2017 Phys. Plasmas 24042508

[18] Deng G et al 2017 Plasma Sci. Technol. 19045101

[19] Xia T Y et al 2017 Nucl. Fusion 57116016 
[20] Chen B et al 2017 Nucl. Fusion 57116025

[21] Olsen J et al 2018 Plasma Phys. Control. Fusion 60085018

[22] Chang C S et al 2017 Nucl. Fusion 57116023

[23] Faisch M et al 2015 Plasma Phys. Control. Fusion 57075005

[24] Xu X Q et al 2010 Phys. Rev. Lett. 105175005

[25] Dudson B D et al 2009 Comput. Phys. Commun. 180 1467-1480

[26] Xu J C et al 2016 Rev. Sci. Instrum. 87083504

[27] Wan B N et al 2015 Nucl. Fusion 55104015

[28] Wan B N et al 2017 Nucl. Fusion 57102019

[29] Luo G-N et al 2017 Nucl. Fusion 57065001

[30] Xu H et al 2018 IEEE Transactions on Plasma Science 465

[31] Li J et al 2013 Nat. Phys. 9 817-821

[32] Gong X et al 2017 59th Annual Meeting of the APS Division of Plasma Physics (Milwaukee, USA) 6212

[33] Ming T F et al 2009 Fusion Engineering and Design 84 57-63

[34] Feng W et al 2017 Nucl. Fusion 57126054

[35] Liang Y et al 2013 Phys. Rev. Lett. 110235002

[36] Horacek J et al 2016 Plasma Phys. Control. Fusion 58074005

[37] Garbet X et al 2004 Plasma Phys. Control. Fusion 46 1351-1373

[38] Herrmann A et al 2007 J. Nucl. Mater. 363-365 528-533

[39] Harrison J R et al 2013 J. Nucl. Mater. 438 S375-S378

[40] Kallenbach A et al 2008 Nucl. Fusion 48085008

[41] LaBombard B et al 2011 Phys. Plasmas 18056104

[42] Wang L et al 2012 Nucl. Fusion 52063024

[43] Petrie T W et al 2013 Nucl. Fusion 53113024

[44] Brunner D et al 2018 Nucl. Fusion 58094002

[45] Sieglin B et al 2013 Plasma Phys. Control. Fusion 55124039

[46] Scarabosio A et al 2015 J. Nucl. Mater. 463 49-54

[47] Eich T et al 2013 J. Nucl. Mater. 438 72-77

[48] LaBombard B et al 2004 Nucl. Fusion 44 1047-1066

[49] Liu J B et al 2016 Nucl. Fusion 56066006 